\title{
Nonlinear Oscillations of an Elastic Inverted Pendulum
}

\author{
Grzegorz Litak \\ Department of Applied Mechanics, \\ Lublin University of Technology, PL-20-618 Lublin, Poland \\ Email: g.litak@pollub.pl, http://litak.pollub.pl
}

\author{
Mattia Coccolo \\ Nonlinear Dynamics, Chaos and Complex Systems Group \\ Departamento de Fisica, Universidad Rey Juan Carlos, \\ Tulipan s/n, 28933 Mostoles, Madrid, Spain
}

\section{Michael I. Friswell, Shaikh F. Ali, Sondipon Adhikari, Arthur W. Lees, Onur Bilgen \\ College of Engineering, Swansea University, Swansea SA2 8PP, United Kingdom}

\begin{abstract}
We examine the dynamics of the elastic inverted pendulum with a tip mass under horizontal harmonic excitation. In particular, we study the conditions when the potential barrier is overcome by the tip mass, and large amplitude oscillations occur, by means of the Melnikov criterion. The results have been confirmed by numerical simulations.
\end{abstract}

\section{INTRODUCTION}

Dymanical behavior of elastic inverted pendulum with a tip mass on one side and other side fixed to the moving base has been analyzed for many years [1], [2], [3], [4]

Recently a larger interest has been focused on beam-mass systems by the occasion of the broadband vibration energy harvester [5], [6]. A new model of an inverted beam coupled to piezoelectric transducer were proposed [7], [8]. The inverted beam has a tip mass such that vertical position is unstable and the beam buckles, giving a double well potential due to gravitational loading. The simulations used harmonic [7] and also stochastic [8] kinematic forcing. In this system, due to nonlinearities of a buckled beam, Friswell et al. [7] showed a multiple solution response, which was presented on the bifurcation diagram. In the present note we discuss that feature by means of the Melnikov approach and basins of attraction. In our case we study a simplified model with a single degreeof-freedom and with a linear effective damping term as in [1], [2], [3].

\section{THE MODEL AND EQUATIONS}

Our simplified model equations and the parameters are based on [1] and our previous papers [7], [8]:

$$
\begin{aligned}
& \left(\alpha_{1}+\alpha_{2} x^{2}\right) \ddot{x}+\alpha_{2} x \dot{x}^{2}+\left(\gamma_{1}+\gamma_{2} x^{2}\right) x+\beta \dot{x} \\
& =\omega^{2} \delta \cos (\omega t),
\end{aligned}
$$

where $x$ is the horizontal displacement of the tip mass and $\dot{x}$ is the corresponding velocity. The set of system parameters considered in the above equations are $\alpha_{1}=0.0294 \mathrm{~kg}$, $\alpha_{2}=1.1094 \mathrm{~kg} / \mathrm{m}^{2}, \gamma_{1}=-0.0633 \mathrm{~N} / \mathrm{m}, \gamma_{2}=53.8479 \mathrm{~N} / \mathrm{m}^{3}$, $\beta=0.01 \mathrm{~kg} / \mathrm{s}, \delta=0.003 \mathrm{~m} \mathrm{~kg}$. The physical meaning of these parameters arises from a Galerkin approximation [1], [3], [7], where the deflection model is assumed to be

$$
\psi(y)=1-\cos \left(\frac{\pi y}{2 L}\right) .
$$

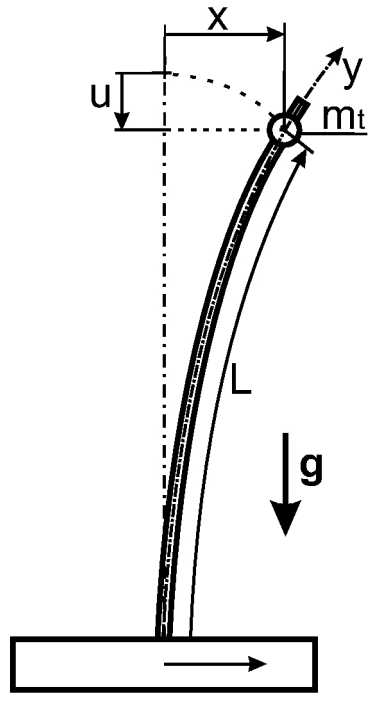

Fig. 1. Schematic plot of an inverted pendulum [8]. See Table 1 for the description and parameter values used.

The system parameters which appear in Eq. (1) are defined as [7]

$$
\begin{aligned}
& \alpha_{1}=N_{5}^{2} I_{0}+M_{t}+\rho A N_{1} \\
& \alpha_{2}=\rho A N_{3}+M_{t} N_{4}^{2}+N_{5}^{4} I_{0}, \\
& \gamma_{1}=E_{s} I_{s} N_{6}-N_{9} \rho A g-N_{4} M_{t} g \\
& \gamma_{2}=2 E_{s} I_{s} N_{7} \\
& \delta=\left(\rho A N_{2}+M_{t}\right) y_{0} .
\end{aligned}
$$

where, after some lengthly mathematical manipulations ([1], [3], [7], [8]), we obtain the constants $N_{1}$ to $N_{9}$ as

$$
\begin{aligned}
& N_{1}=\int_{0}^{L} \psi(y)^{2} \mathrm{~d} y=L\left(\frac{3}{2}-\frac{4}{\pi}\right), \\
& N_{2}=\int_{0}^{L} \psi(y) \mathrm{d} y=\frac{L}{\pi}(\pi-2), \\
& N_{3}=\int_{0}^{L}\left(\int_{0}^{y} \psi^{\prime}(x)^{2} \mathrm{~d} y\right)^{2} \mathrm{~d} y=\frac{\pi^{2}}{384 L}\left(2 \pi^{2}-9\right), \\
& N_{4}=\int_{0}^{L} \psi^{\prime}(y)^{2} \mathrm{~d} y=\frac{\pi^{2}}{8 L},
\end{aligned}
$$


TABLE I

SYSTEM PARAMETERS

\begin{tabular}{|l|l|}
\hline symbol and value & description \\
\hline$L=0.2 \mathrm{~m}$ & length of the beam \\
$m_{t}=0.038 \mathrm{~kg}$ & tip mass \\
$\rho=7850 \mathrm{~kg} / \mathrm{m}^{3}$ & density of the beam mass \\
$b_{b}=15 \mathrm{~mm}$ & beam width \\
$h_{b}=2.5 \mathrm{~mm}$ & beam thickness \\
$A=b_{b} h_{b}=0.000004 \mathrm{~m}^{2}$ & cross section area of beam \\
$E=210 \mathrm{GPa}$ & Young's modulus \\
$I=2.15 \times 10^{-14} \mathrm{~m}^{4}$ & geometrical moment of inertia \\
$I_{0}=1144.4 \times 10^{-3} \mathrm{kgm}^{2}$ & mass moment of inertia \\
$y=y_{0} \cos (\omega t)$ & kinematic displacement of the base \\
\hline
\end{tabular}

$$
\begin{aligned}
& N_{5}=\psi^{\prime}(L)=\frac{\pi}{2 L}, \\
& N_{6}=\int_{0}^{L} \psi^{\prime \prime}(y)^{2} \mathrm{~d} x=\frac{\pi^{4}}{32 L^{3}}, \\
& N_{7}=\int_{0}^{L} \psi^{\prime \prime}(y)^{2} \psi^{\prime}(y)^{2} \mathrm{~d} x=\frac{\pi^{6}}{512 L^{5}}, \\
& N_{8}=\int_{0}^{L} \psi^{\prime \prime}(y)^{2} \psi^{\prime}(y)^{4} \mathrm{~d} x=\frac{\pi^{8}}{4096 L^{7}}, \\
& N_{9}=\int_{0}^{L}\left(\int_{0}^{y} \psi^{\prime}\left(y^{2} \mathrm{~d} y\right) \mathrm{d} x=\frac{1}{16}\left(\pi^{2}-4\right),\right.
\end{aligned}
$$

where $\psi^{\prime}(x)$ is the derivative $\mathrm{d} \psi(x) / \mathrm{d} x$.

\section{MELNiKOV APPROACH AND SIMULATION RESUlTS}

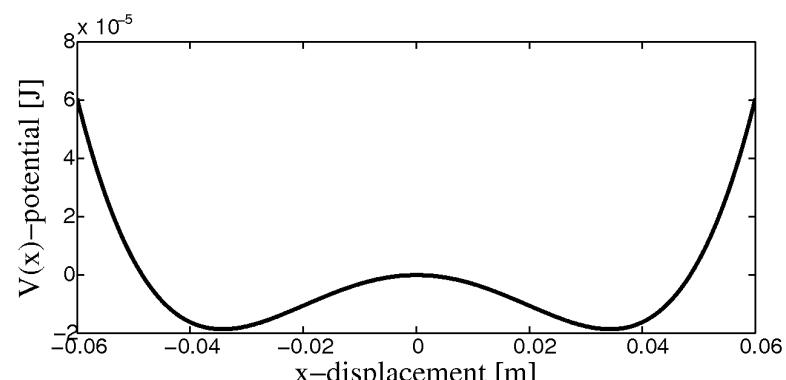

(a)

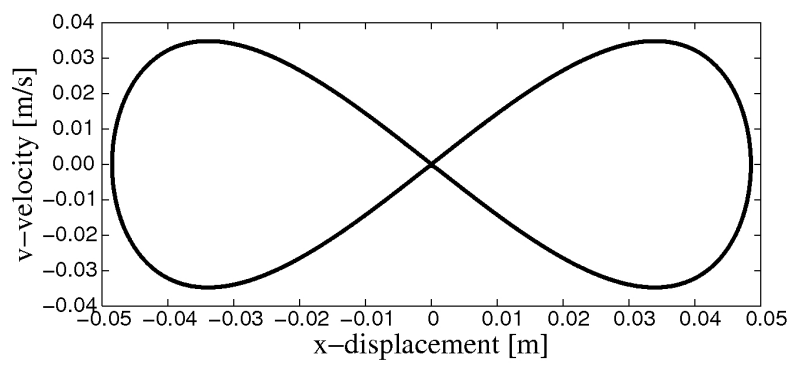

(b)

Fig. 2. (a) Potential of the undisturbed Hamiltonian system. (b) Two homoclinic trajectories (see the left and right sides of the saddle point $\left.\left(x_{0}, v_{0}\right)=(0,0)\right)$.

The Melnikov analysis predicts the conditions of escape from a single potential well. In this method the excitation and damping terms in the original system (Eq. (1)) are treated as perturbations to the homoclinic orbits. The conditions for the system to escape from the potential well, or the evidence of fractalization of the basins of attraction, are signaled by

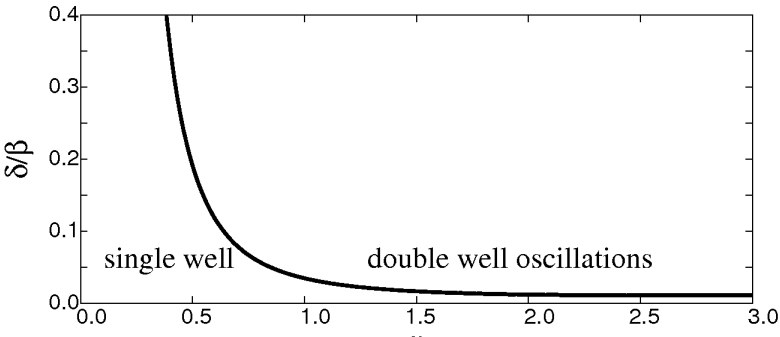

(a)

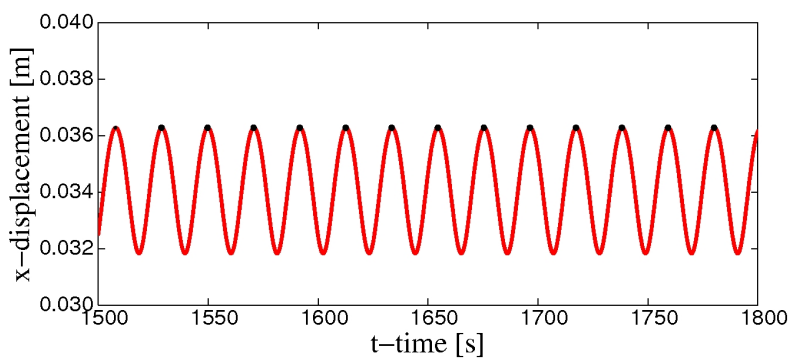

(b)

(c)

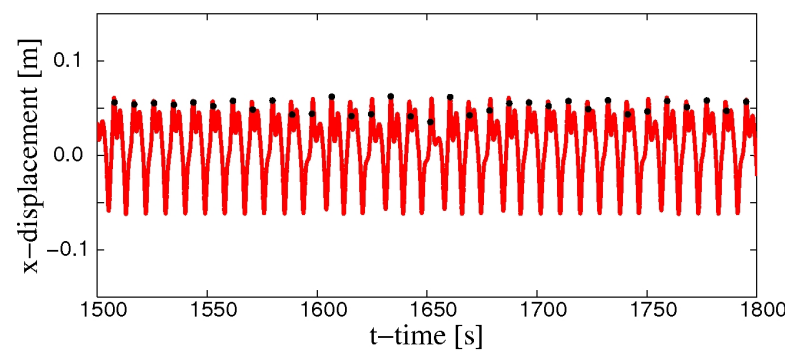

(d)

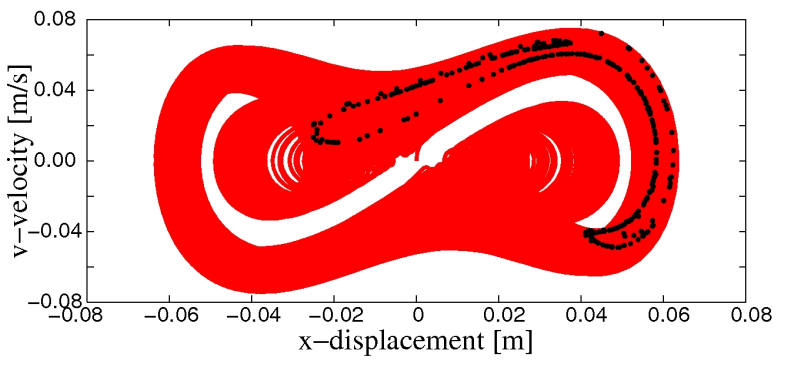

Fig. 3. (a) Critical values of the ratio $(\delta / \beta)$. (b), (c) Time histories of the tip mass displacement for excitation frequencies $\omega=0.3$ and $1.2 \mathrm{rad} / \mathrm{s}$, respectively. (d) The phase portrait of the system response for the excitation frequency $\omega=0.7$. The black points in (b)-(d) correspond to Poincaré stroboscopic points. The initial conditions were $(x(0), \dot{x}(0))=(0,0)$.

the cross-sections of the stable and unstable manifolds [9]. Such a condition can be expressed in terms of the ratio of the excitation amplitude to the damping coefficient $(\delta / \beta)$.

The Hamiltonian system is obtained in the limit $\delta, \beta \rightarrow 0$ as

$$
\left(\alpha_{1}+\alpha_{2} x^{2}\right) \ddot{x}+\alpha_{2} x \dot{x}^{2}+\left(\gamma_{1}+\gamma_{2} x^{2}\right) x=0 .
$$

For this system Fig. 2 shows the potential and homoclinic orbits.

The critical value $\delta / \beta$ has been estimated numerically and plotted in Fig. 3(a). Figures 3(b) and (c) illustrate the system evolution with changing $\omega$, as shown by the time series. Note that Fig 3(b) represents a regular type of oscillation limited to one of the potential wells, while Fig. 3(c) shows the non- 
periodic response of the examined system extending to both potential wells. Fig. 3(d) shows the phase portrait for this case which represents a chaotic attractor (the black points correspond to the Poincare section). The dynamics of the above system, where the potential barrier is overcome, could be used to improve the performance of broadband energy piezoelectric harvesters [7].

\section{BASINS OF ATTRACTION AND WADA REGIONS}

In nonlinear systems the basins of attraction are defined by subsets of the state space to which trajectories originating from different initial conditions tend during time evolution. In a double well Duffing-like system (Eq. (1)) we have, normally, two attractors in each of the potential wells. The external forcing creates a third basin of orbits which escape from the potential wells.

In fact, for each such attractor, its basin of attraction is the set of initial conditions leading to long-time behavior that approaches that attractor. Thus the qualitative behavior of the long-time motion of a given system can be fundamentally different depending on which basin of attraction the initial condition lies in (e.g., attractors can correspond to periodic, quasi-periodic or chaotic behaviors of different types).

We decided to calculate the basin of attraction through an algorithm that evaluates the distance, using a Euclidean metric, between the long term behavior of the trajectories and the attractor. In the case where we have multiple destinations for the scattering trajectories, the structure of the basins can, eventually, be more complicated and shows Wada properties [10]. A basin exhibits the Wada property if any boundary point also belongs to the boundary of two other basins. More specifically, an open neighborhood of a point $\left(x_{0}, \dot{x}_{0}\right)$ belonging to a Wada basin has a non empty intersection with at least three different basins. In this case the dynamics of the system becomes even more unpredictable.

Using our dynamical system defined in Eq. (1) we performed simulations and the results are presented in Fig. 4. One can see that all the three attractors are forming a complex Wada structure.

\section{CONCLUSIONS}

In this note we investigated the dynamical system of an inverted pendulum-like beam with a tip mass excited horizontally. In this system we investigated Melnikov chaos for fractal borders between basins of attraction and stationary chaos with typical strange attractors' appearance. Finally we investigated a creation of Wada patterns with complex mixtures of three basins of attraction (see three colours in Fig. 4). The Wada regions have a multifractal structure. As expected increasing the excitation term, $\delta$, leads to a larger percentage of white regions which (Fig. 4) indicates the escape phenomenon.

\section{ACKNOWLEDGMENT}

The authors gratefully acknowledge the support of the Royal Society through International Joint Project No. JP090343. GL thanks for the support of the 7th Framework Programme FP7REGPOT-2009-1, under Grant Agreement No. 245479.
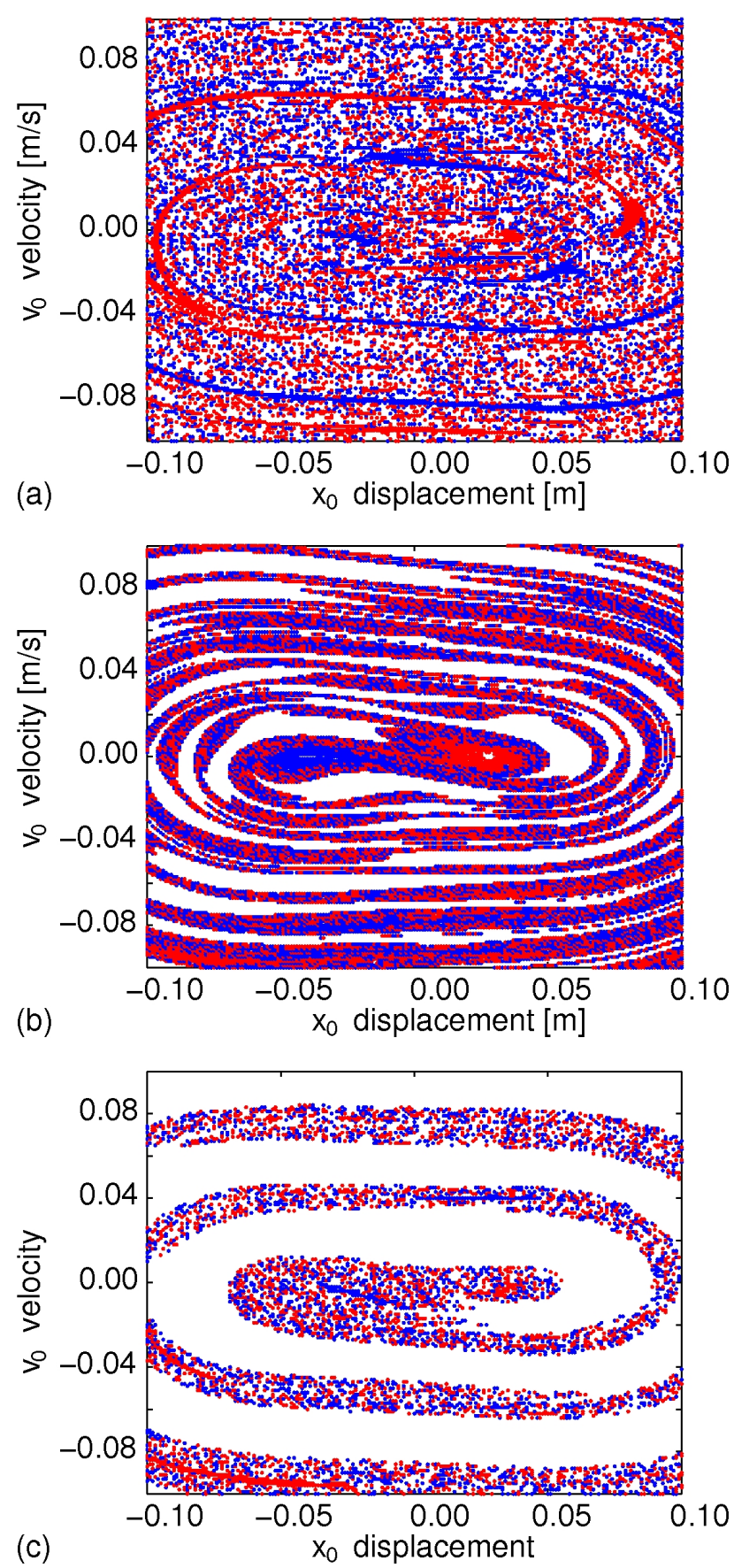

Fig. 4. Basins of attraction for different system parameters $\omega=0.03$ $\delta=0.003$ (a) $\omega=0.8, \delta=0.005$ (b) $; \omega=0.3, \delta=0.008$ (here $\beta=0.01$ for al cases) Red and blue colours indicate the basins for single well oscillations while the white region is related to the escape phenomenon (either regular or chaotic).

\section{REFERENCES}

[1] L.D. Zavodney, A.H. Nayfeh, "The nonlinear response of a slender bean carrying lumped mass to a principal parametric excitation: theory and experiment", Int. J. Non-Linear Mech. 24, pp. 105-125, 1989.

[2] A. Nayfeh and P. Pai, Linear and Nonlinear Structural Mechanics, Wiley Interscience, New Jersey 2004.

[3] E. Esmailzadeh, G. Nakhaie-Jazar, "Periodic behavior of a cantilever 
beam with end mass subjected to harmonic base excitation", textitInt. J. Non-linear Mech., 33, pp. 567-577, 1998.

[4] S.F. Ali and R. Padhi, "Active vibration suppression of non-linear beams using optimal dynamic inversion”, P.I.Mech.Eng. I-J. Sys. Contr. Eng. 223, pp. 657-672, 2009.

[5] A. Erturk, D. Inman, Piezoelectric Energy Harvesting, Wiley, Chichester 2011.

[6] L. Tang, Y. Yang, C.K. Soh, "Toward broadband vibration-based energy harvesting", J. Int. Mat. Syst. Struc. 21, 1867-1897 (2010).

[7] M.I. Friswell, S.F. Ali, S. Adhikari, A.W. Lees, O. Bilgen, G. Litak, "Nonlinear piezoelectric vibration energy harvesting from an inverted cantilever beam with tip mass", J. Int. Mat. Syst. Struc. 2012, doi:10.1177/1045389X12455722.

[8] M. Borowiec, G. Litak, M.I. Friswell, S.F. Ali, S. Adhikari, A.W. Lees and O. Bilgen, "Energy harvesing in piezoelastic systems driven by random excitations", Int. J. Struc. Stab. Dynamics 2012 in press.

[9] L. Ruzziconi, G. Litak, S. Lenci, "Nonlinear oscillations, transition to chaos and escape in the duffing system with non-classical damping", Journal of Vibroengineering 13, pp. 22-38, 2011.

[10] J. Aguirre, J.C. Vallejo and, M.A.F. Sanjuan, "Wada basins and chaotic invariant sets in the Henon-Heiles system", Phys. Rev. E, 64, 066208, 2001 . 